\title{
Transabdominal bowel sonography for the detection of intestinal complications in Crohn's disease
}

C Gasche, G Moser, K Turetschek, E Schober, P Moeschl, G Oberhuber

\begin{abstract}
Background-The course of Crohn's disease is characterised by the occurrence of intestinal complications such as strictures, intra-abdominal fistulas, or abscesses. Standard diagnostic procedures may fail to show these complications, in particular fistulas.

Aims-To test the value of transabdominal bowel sonography (TABS) for the detection of intestinal complications in Crohn's disease.

Methods-TABS was prospectively performed in 213 patients with Crohn's disease in a university based inflammatory bowel disease referral centre. Thirty three underwent resective bowel surgery and were included in this study. The accuracy of TABS to detect strictures, intraabdominal fistulas, or abscesses was compared with surgical and pathological findings.

Results-TABS was able to identify strictures in 22/22 patients and to exclude it in $10 / 11$ patients $(100 \%$ sensitivity, $91 \%$ specificity). Fistulas were correctly identified in 20/23 patients and excluded in 9/10 patients ( $87 \%$ sensitivity, $90 \%$ specificity). Intra-abdominal abscesses were correctly detected in 9/9 patients and excluded in $22 / 24$ patients $(100 \%$ sensitivity, $92 \%$ specificity).

Conclusions-In experienced hands TABS is an accurate method for the detection of intestinal complications in Crohn's disease. TABS is thus recommended as a primary investigative method for evaluation of severe Crohn's disease.

(Gut 1999;44:112-117)
\end{abstract}

Keywords: Crohn's disease complications; fistula; stricture; abscess; bowel sonography

The chronic course of Crohn's disease is characterised by the occurrence of intestinal complications, namely strictures, fistulas, or abscesses. ${ }^{12}$ As the therapeutic management of these patients usually changes with the incidence of a complication, ${ }^{3}{ }^{4}$ sensitive diagnostic tools are needed. Standard diagnostic procedures such as ileocoloscopy or barium studies can reliably visualise bowel obstruction. These techniques, however, are of limited usefulness with regard to the detection of small enteromesenteric fistulas and usually fail to show abscesses. ${ }^{5}$ Granulocyte scintigraphy and computed tomography have been reported to be of value in the detection of the latter lesions. ${ }^{6-10}$ The urgent and routine availability of granulocyte scintigraphy, however, is limited. Small intraperitoneal abscesses may also remain unidentified by computed tomography. ${ }^{71}$ Magnetic resonance imaging is now routinely used to visualise perianal disease, ${ }^{12}{ }^{13}$ but its ability to show intra-abdominal lesions has not yet been defined. ${ }^{14}{ }^{15}$ Although these various methods are available and frequently used, the detection of intra-abdominal enteromesenteric or small enteroenteric fistulas remains a serious clinical problem. It is not unusual, therefore, that previously unrecognised fistulas are found at the pathological work up of resected bowel specimens.

Transabdominal bowel sonography (TABS) can visualise and locate transmural bowel inflammation with alteration of the echoarchitecture of the bowel wall in Crohn's disease. ${ }^{16-21}$ It has been reported that this method is useful in diagnosing Crohn's disease, ${ }^{22}$ in the differential diagnosis of ulcerative colitis, ${ }^{23-25}$ and in the determination of the extent of disease. ${ }^{26}$ In the past decade technical developments in sonographic devices improved image resolution. In parallel, experience of investigators increased and resulted in higher sensitivity of TABS. TABS is a non-invasive, inexpensive, radiation free method that is well tolerated by patients. Repeated investigations can be easily performed during the clinical follow up. In this prospective study we investigated the validity of TABS in the detection of disease specific intestinal complications in Crohn's disease. Both surgery and pathology findings were used as reliable control methods.

\section{Patients and methods}

PATIENTS

Between July 1994 and September 1996 TABS was repeatedly performed in 213 patients with Crohn's disease in the outpatient clinic of an inflammatory bowel disease referral centre. The following patients were excluded: those with clinically obvious fistulas such as in the enterourinary or enterocutaneous tracts; and those who had had bowel resection within six months in order to avoid interference with surgery associated complications. Of the 213 patients, 33 underwent resective bowel surgery and were further evaluated in this study. The decision to operate was taken independently of the TABS findings on the basis of clinical symptoms, laboratory results, and imaging studies by a sovereign physician.

Abbreviations used in this paper: TABS, transabdominal bowel sonography. 
The median age of the 33 patients was 31 years (range 18-51 years). There were 19 women and 14 men. The resection was the first in 21 patients, the second in seven, the third in four, and the fifth in one. One patient had a colostomy. Eleven patients received steroids, 13 received mesalazine, three were on azathioprine, and 10 did not receive any medication. The clinical activity ranged between two and 14 points (median seven points) measured by means of the Harvey-Bradshaw index. ${ }^{27}$ The median period between TABS and surgery was 24 days (range $1-132$ days).

The 180 patients who did not undergo surgery were not evaluated further in this study. In 12 of these 180 patients TABS showed an intestinal complication (nine strictures with additional fistulas in two patients, and three fistulas alone). All strictures and one of five fistulas were confirmed by small bowel barium studies. TABS did not show any complication in $168 / 180$ patients. Retrospective analysis of endoscopic or radiological studies performed within six months before or after TABS revealed bowel obstruction in five and fistulas or abscesses in none of these patients. These patients $(n=17)$ did not undergo resective surgery because of the following reasons: lack of clinical symptoms $(n=10)$, diffuse extent of disease $(n=5)$, unwilling to undergo surgery $(\mathrm{n}=1)$, and short bowel syndrome $(n=1)$.

TRANSABDOMINAL BOWEL SONOGRAPHY

Real time TABS was performed using 3.5 $\mathrm{MHz}, 78 \mathrm{~mm}$ curved array and $10-5 \mathrm{MHz}$ broadband, $38 \mathrm{~mm}$ linear array transducers (Ultramark 9, Advanced Technology Laboratories Inc., Bothel, Washington, USA). TABS was done in the morning about two to five hours after breakfast. No special preparation such as overnight fasting, oral deflating drugs, or water enema were prescribed. The duration of investigation ranged from 10 to 30 minutes. The investigators were aware of the diagnosis, previous bowel resections, and the current clinical symptoms but were blinded to the results of other morphological investigations. The morphology and location of bowel wall thickening, mesenteric masses, intraabdominal fistulas, abscesses, or strictures were documented. No evaluation of the stomach or the distal (deep pelvic) part of the sigmoid and rectum was intended.

\section{Morphology of complications in TABS}

Bowel strictures were assumed in the case of severe luminal narrowing in regions of bowel wall thickening (at least $3 \mathrm{~mm}$ ), accompanied by destruction of wall layering and loss of peristaltic bowel movement with or without prestenotic bowel dilatation. In areas of bowel wall thickening hypoechoic peri-intestinal lesions were regarded as fistulas when their cross sectional diameter was below $2.0 \mathrm{~cm}$ (arbitrary cut off point). Similar lesions equal or above 2.0 $\mathrm{cm}$ were considered to be abscesses.
SURGERY AND PATHOLOGY

The results obtained from surgery and pathology were taken as standard. Both surgeon and pathologist were blinded to the result of the preoperative TABS. Bowel resection specimens were fixed in $4 \%$ buffered formalin. In gross examination the whole bowel was cut into circumferential cross sections $0.5 \mathrm{~cm}$ in thickness in order to reveal the structure of the bowel wall and mesentery and to detect fistulas and strictures. Areas of interest (fistulas, strictures, areas of inflammation) were examined by histology after staining $5 \mu \mathrm{m}$ thick sections with haematoxylin and eosin. Bowel obstruction was diagnosed in cases of luminal narrowing and bowel wall thickening with or without prestenotic dilatation by naked eye examination. Fistulas were defined as abnormal communications between the lumen of the gut and the mesentery and/or another hollow organ. All lesions that had penetrated the muscularis propria were considered to be fistulas. They were characterised by a central lumen containing leucocytes and necrotic debris with a border of granulation tissue. Abscesses were defined as intraperitoneal or mesenteric collections of fluid containing necrotic debris and leucocytes with a diameter of more than $1.0 \mathrm{~cm}$.

\section{Results}

DETECTION OF COMPLICATIONS BY TABS

Of the 33 patients who underwent surgery, preoperative TABS revealed at least one intraabdominal complication in 32 patients. A single patient had no complication recognised by TABS. In $70 \%$ of patients TABS showed strictures $(n=23)$, which were located in the small bowel in 18 (including the ileocaecal valve or anastomosis) and in the colon in five. Of these 23 patients, 11 were considered to have strictures alone (example shown in fig 1B), 12 showed peri-intestinal lesions which were considered to be fistulas (example shown in fig 2A), and in four patients TABS was consistent with the presence of an additional abscess. Another nine patients were considered to have fistulas without strictures and with an additional abscess in seven (example shown in fig 3).

SURGICAL AND PATHOLOGICAL FINDINGS

Sixteen patients underwent small bowel resection, five colonic resection, and 12 ileocolonic resection. A median of $30 \mathrm{~cm}$ of small bowel (range $5-100 \mathrm{~cm}$ ) and $16 \mathrm{~cm}$ of colon (3-65 $\mathrm{cm}$ ) was resected. Surgical and pathological evaluations revealed a total of 54 complications occurring in 31 patients (table 1). Sixty seven per cent of patients had strictures either alone $(n=8)$ or in combination with fistulas $(n=11$; example shown in fig $2 \mathrm{~B})$ and abscesses $(n=3)$. Twenty seven per cent had fistulas alone $(n=3)$ or combined with abscesses $(n=6)$. In two patients no intestinal complication was detected. The anatomy of fistulas was enteroenteric in five, enteromesenteric in 16, and both in two patients. Abscesses did not occur without coexisting fistulas. 


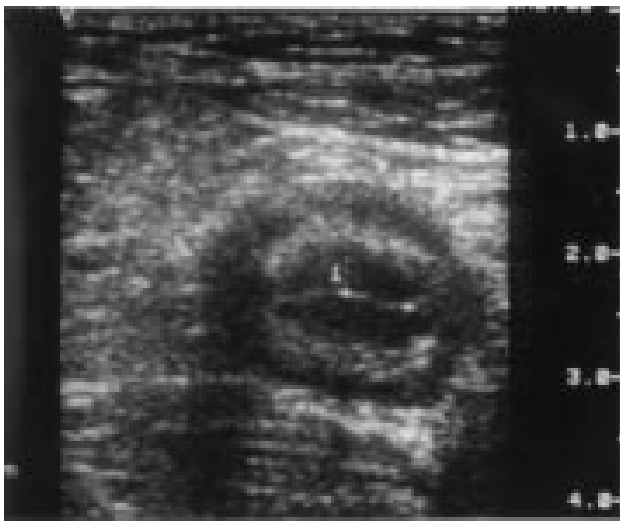

A

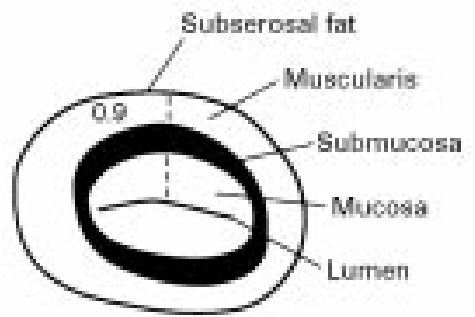

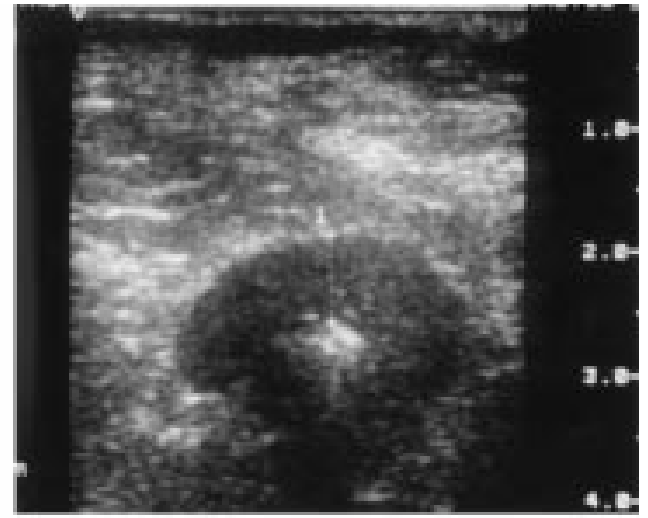

B

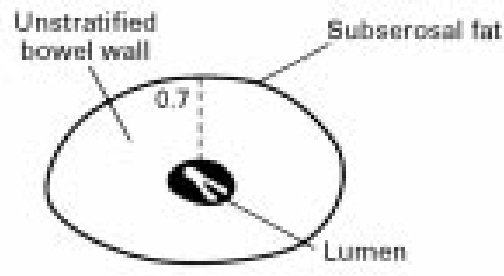

Figure 1 Transverse TABS section (using a 10-5 MHz array) through the terminal ileum of two patients, five years ( $A$ ) and eight years (B) after ileocaecal resection. Both patients show significant bowel wall thickening, $0.9 \mathrm{~cm}(A)$ and $0.7 \mathrm{~cm}$ (B). In (A), note the four layers of a typical target lesion and the hyperechoic, linear shaped collapsed lumen of the gut. No significant luminal narrowing and no other complications are present. In (B), note the loss of stratification of the bowel wall echo pattern reflecting destruction of layers after long term inflammation. The central hyperechoic, point shaped lumen corresponds to a stricture.
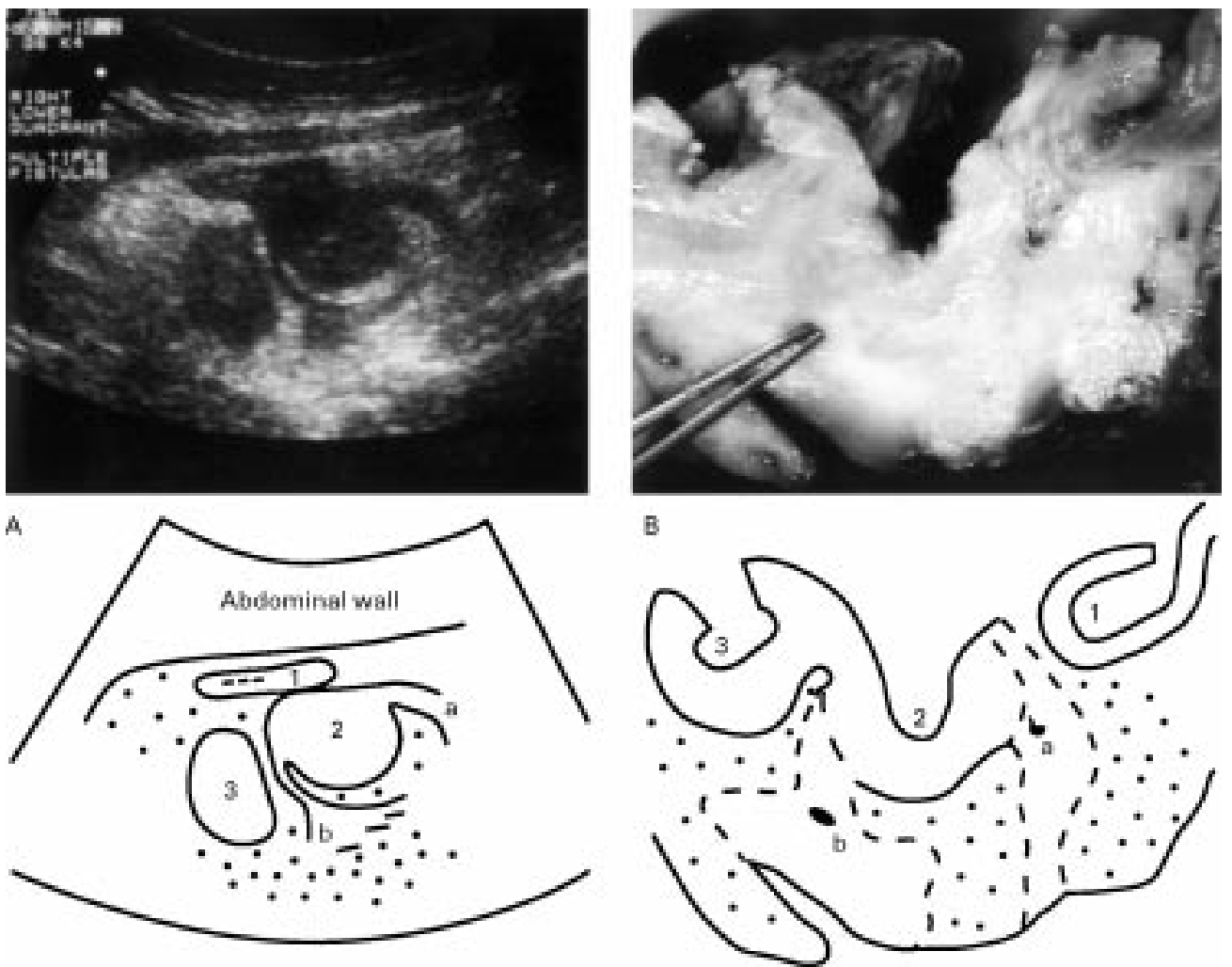

Figure 2 (A) Transverse TABS section (3.5 MHz array) through the right lower quadrant of a patient with Crohn's disease. Three adherent bowel loops of the terminal ileum (1,2,3, from oral to anal) are shown with hypoechoic peri-intestinal lesions $(a, b)$ arising from loop 2 and hyperechoic wrapping mesentery (dotted area). Loop 1 is collapsed with only little thickening of the bowel wall $(0.4 \mathrm{~cm})$. Loops 2 and 3 show severe thickening of the bowel wall $(1.2 \mathrm{~cm}$ and $1.0 \mathrm{~cm}$ ), loss of bowel wall layering, and no detectable luminal hyperechoic content. These findings were regarded as high grade bowel obstruction of loops 2 and 3 with fistulas arising from loop 2. (B) Transverse section through the corresponding resection specimen after overnight fixation, longitudinal incision of the lumen, and unfolding of bowel loops (1, 2, 3, according to $A$ ) which resulted in rearrangement of loop 1 . The forceps point into one of two fistulous tracts ( $a, b$, according to A). Note perifistulous fibrotic tissue (broken lines) surrounded by fibrofatty proliferation of the mesentery (dotted area). 

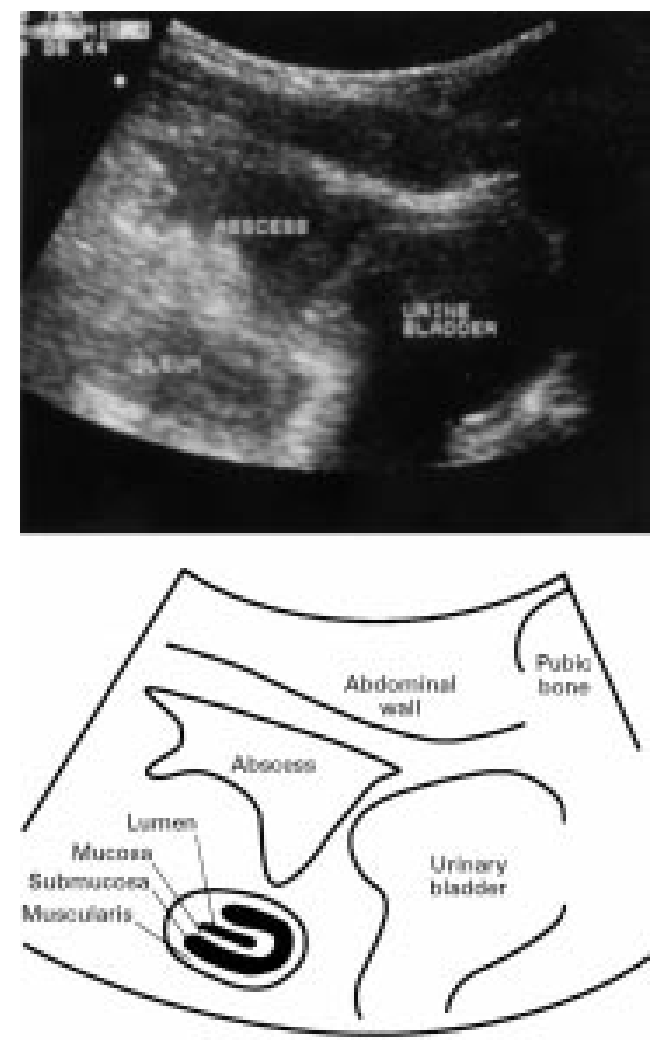

Figure 3 Sagittal TABS section through the mid lower abdomen of a patient with a two year history of uncomplicated Crohn's disease. A $4 \mathrm{~cm}$ hypoechoic peritoneal lesion (abscess) is present cranial to the urinary bladder. The adjacent ileum shows wall thickening (about $0.7 \mathrm{~cm}$ ) with typical layering and a hyperechoic linear shaped central lumen without evidence of stricturing. The abscess originates from an ileal fistula (not visible on this section).

Table 1 Results of surgical and pathological evaluation

\begin{tabular}{ll}
\hline & No $(\%)$ \\
\hline Stricture & $8(24.2)$ \\
Stricture and fistula & $11(33.3)$ \\
Stricture, fistula and abscess & $3(9.1)$ \\
Fistula & $3(9.1)$ \\
Fistula and abscess & $6(18.2)$ \\
No complication & $2(6.1)$ \\
Total & $33(100.0)$ \\
\hline
\end{tabular}

COMPARISON OF TABS WITH SURGICAL AND PATHOLOGICAL FINDINGS

It was possible to identify bowel obstruction in $22 / 22$ patients (sensitivity $100 \%$ ) and to exclude it in $10 / 11$ patients (specificity $91 \%$ ) by TABS (see table 2). Fistulas were truly identified in $20 / 23$ patients (sensitivity $87 \%$ ) and excluded in $9 / 10$ patients (specificity $90 \%$ ). Intra-abdominal abscesses were correctly detected in $9 / 9$ patients (sensitivity

Table 2 Sensitivity and specificity of transabdominal bowel sonography (TABS) in the detection of intestinal complications

\begin{tabular}{|c|c|c|c|c|c|c|}
\hline \multirow[b]{2}{*}{$\begin{array}{l}\text { Surgery and } \\
\text { pathology }\end{array}$} & \multicolumn{4}{|c|}{ Results of TABS } & \multirow[b]{2}{*}{$\begin{array}{l}\text { Sensitivity } \\
(\%)\end{array}$} & \multirow[b]{2}{*}{$\begin{array}{l}\text { Specificity } \\
(\%)\end{array}$} \\
\hline & $\begin{array}{l}\text { True } \\
\text { positive }\end{array}$ & $\begin{array}{l}\text { True } \\
\text { negative }\end{array}$ & $\begin{array}{l}\text { False } \\
\text { positive }\end{array}$ & $\begin{array}{l}\text { False } \\
\text { negative }\end{array}$ & & \\
\hline Stricture $(n=22)$ & 22 & 10 & 1 & 0 & 100 & 91 \\
\hline Fistula $(n=23)$ & 20 & 9 & 1 & 3 & 87 & 90 \\
\hline Abscess $(n=9)$ & 9 & 22 & 2 & 0 & 100 & 92 \\
\hline Total $(n=54)$ & 51 & 41 & 4 & 3 & 94 & 91 \\
\hline
\end{tabular}

$100 \%)$ and excluded in $22 / 24$ patients $(92 \%$ specificity).

\section{Discussion}

The accurate detection of intestinal complications is a major issue in the management of patients with Crohn's disease. In this prospective study we evaluated the ability of TABS to detect strictures, fistulas, or intra-abdominal abscesses. Our results show that TABS can visualise strictures and intra-abdominal abscesses with high accuracy. Furthermore, TABS had impressive sensitivity with regard to detection of fistulas: only $3 / 23$ of these lesions were not detected using TABS.

In another prospective study which tested the ability of TABS to detect intestinal complications in Crohn's disease, Maconi et a ${ }^{28}$ compared the results from TABS with colonoscopy, small bowel $x$ ray, barium enema, and computed tomography. They reported comparable results concerning the detection of strictures (74\% sensitivity) and abscesses (83\% sensitivity). Intra-abdominal fistulas, however, were discovered in only $50 \%$. This is in disagreement with our results. However, there are two important variations in the design of the two studies: the definition of fistulas; and the use of different standards.

With respect to the first point, fistulas are generally regarded as tracts that connect two epithelial surfaces. ${ }^{29}$ It has been proposed that fistulas in Crohn's disease arise from fissures that may progress to sinus tracts which have already penetrated through the muscle coat into the mesentery. ${ }^{30}$ They form small abscesses and may then track through the wall of an adjacent hollow organ (usually another bowel loop ${ }^{31}$ ). Other investigators, including Maconi et al, based their definition of fistulisation on the radiological appearance of the connecting tract. In contrast we classified a lesion to be a fistula when it had fully penetrated the bowel wall. Thus, in our study both blind ending sinus tracts and connecting tracts were regarded as fistulas. We consider the event of bowel penetration to be relevant for clinical practice as this process carries the risk of bacterial complications in Crohn's disease, such as abscess formation, peritonitis, or sepsis, which may ultimately lead to death. ${ }^{3}{ }^{4}$ Both studies vary not only in the pathological aspect of fistulisation but also in its sonographic appearance: Maconi et al considered fistulas as hypoechoic duct-like structures between bowel loops containing fluid or air and which may pass echogenic material. We suppose that this kind of definition has derived more from the radiological point of view than from the pathophysiological mechanisms of the underlying disease. Our definition of fistulas by TABS was a hypoechoic periintestinal lesion arising in areas of bowel wall thickening. Most of these lesions did not contain air and we were never able to document the passage of echogenic material. In these hypoechoic lesions it is not possible to discriminate between the fluid containing small fistulous tract and the adjacent granulation tissue. This is the reason why sonography tends to overestimate the size of fistulas. We have, therefore, 
chosen a sonographic diameter of $2 \mathrm{~cm}$ as the limit between fistulas and abscesses. Frequently there is valuable demarcation between these hypoechoic lesions and the surrounding hyperechoic masses that correspond to fibrofatty proliferation of the mesentery (fig 2). In our experience the identification of such wrapping hyperechoic mesenteric masses calls for a careful sonographic search for fistulas in these areas.

With respect to the use of different standards, fistulas are frequently missed by conventional diagnostic procedures. In these cases an accurate diagnosis of fistulas can only be made during the pathological work up of resected specimens. There are few prospective investigations which have tested the value of barium studies or computed tomography for detection of fistulas in Crohn's disease. ${ }^{32}$ These techniques have not been sufficiently compared with surgical and pathological findings and they are frequently inconsistent concerning the detection of fistulas. ${ }^{10}$ We therefore considered the use of these techniques as standards to be inappropriate and compared TABS with surgery and pathology.

We are aware of the fact that the high prevalence of intestinal complications in our study population incorporates a selection bias. Fistulas were present in $70 \%$, strictures in $67 \%$, and abscesses in $27 \%$ of patients, which is clearly much higher than in the general Crohn's disease population. The high prevalence of complications in our study population is related to overestimation of the positive predictive value. ${ }^{33}$ The retrospective analysis of the 180 non-operated patients showed weaker results concerning the total sensitivity $(67 \%)$ and specificity $(99 \%)$. The positive predictive value for detection of fistulas was as low as $20 \%$. These data not only reflect the inadequacy of barium studies (used as the "gold standard" ${ }^{34}$ ) but may also point to an overestimation of the accuracy of TABS. Thus, the validity of TABS has to be interpreted with caution. Furthermore, we tested TABS in known Crohn's disease patients only. Our findings cannot be extended to patients without previous diagnosis or to other populations with similar intestinal complications such as those with diverticulitis.

The appropriate use of imaging techniques in Crohn's disease depends on the specific goal in the individual patient. Each method has its limitations - for example, it is obvious that small bowel enteroclysis is not suitable for visualisation of sigmoid ulcers. With respect to TABS it is important to recognise its anatomical limitations: the stomach, the deep pelvic part of the sigmoid, and the rectum are difficult to study. We therefore did not attempt to detect complications in these areas. In this series gastric surgery was not performed. There was no case of an isolated distal sigmoid or rectal lesion. However, one of the three unrecognised fistulas was an ileosigmoid tract. This indicates that not only the distal sigmoid but also the deep pelvic part of the ileum may be invisible to TABS. Another limitation of TABS is the differentiation of hypoechoic peri-intestinal lesions in the early postoperative course. TABS fails to distinguish between granulation tissue in areas of previous drainage and fistulous tracts. We thus excluded early postoperative patients from the protocol.

The understanding of both indications and limitations of TABS will enable appropriate use of this method in future. TABS is a safe, inexpensive, non-invasive, and radiation free technique that does not require special preparation. Thus it is highly acceptable to the patients. We recommend TABS to be used as primary investigation in patients with Crohn's disease when intestinal complications are suspected. We speculate that the early recognition of such complications will improve management of the patients and hence their quality of life. In addition, the timely detection of intraabdominal fistulas or abscesses may help to lower the incidence of sepsis and mortality in this young population.

We are indebted to Professor A Gangl for encouraging discussions and critical review of the manuscript, to $\mathrm{T}$ Krutt for generation of the data file, and to S Bakos for delineating the graphs.

1 Farmer RG, Hawk WA, Turnbull RB Jr. Indications for surgery in Crohn's disease. Gastroenterology 1976;71:245-50.

2 Farmer RG, Whelan G, Fazio VW. Long-term follow-up of patients with Crohn's disease. Gastroenterology 1985;88:
$1818-25$.

Ribeiro MB, Greenstein AJ, Yamazaki Y, et al. Intraabdominal abscess in regional enteritis. Ann Surg 1991; 213:32-6.

4 Fishman EK, Wolf EJ, Jones B, et al. CT Evaluation of Crohn's disease: effect on patient management. Am $\mathcal{F}$ Roentgenol 1987; 148:537-40

5 Cybuisky IJ, Tam P. Intra-abdominal abscesses in Crohn's Cybuisky IJ, Tam P. Intra-abdominal
disease. Am $\mathcal{f}$ Surg 1990;56:678-82.

6 Wheeler JG, Slack NF, Duncan A, et al. The diagnosis of intra-abdominal abscesses in patients with severe Crohn's disease. Qf Med 1992;82:159-67.

7 Koikman JJ, Falke THM, Roos JC, et al. Computed tomography and granulocyte scintigraphy in active inflammatory bowel disease: comparison with endoscopy and operative findings. Dig Dis Sci 1996;41:641-50.

8 Goldberg HI, Gore RM, Margulis AR, et al. Computed tomography in the evaluation of Crohn disease. Am 7 Roentgenol 1983;140:277-82.

9 Gore RM, Marn CS, Kirby DF, et al. CT findings in ulcerative, granulomatous, and indeterminate colitis. Am F Roentgenol 1984;143:279-84.

10 Greenstein Orel S, Rubesin SE, Jones B, et al. Computed tomography vs barium studies in the acutely symptomatic patient with Crohn disease. I Comput Assist Tomogr 1987;11:1009-16.

11 Schneekioth G, Terrier F, Fuchs WA. Computed tomography of intraperitoneal abscesses. Gastrointest Radiol 1982;7: 35- 41

12 Skaiej M, Makowiec F, Weintich M, et al. Magnetic resonance imaging in perianal Crohn's disease. Dtsch Med Wochenschr 1993;118:1791-6.

13 Haggett PJ, Moore NR, Shearman JD, et al. Pelvic and perineal complications of Crohn's disease: assessment using magnetic resonance imaging. Gut 1995;36:407-10.

14 Kettritz U, Isaacs K, Warshauer DM, et al. Crohn's disease. Pilot study comparing MRI of the abdomen with clinical evaluation. F Clin Gastroenterol 1995;21:249-53.

15 Madsen SM, Thomsen HS, Munkholm P, et al. Magnetic resonance imaging of Crohn disease: early recognition of treatment response and relapse. Abdom Imaging 1997;22: 164-6.

16 Hott S, Samuel E. Grey scale ultrasound in Crohn's disease. Gut 1979;20:590-5.

17 Sonnenberg A, Erckenbrecht J, Peter P, et al. Detection of Crohn's disease by ultrasound. Gastroenterology 1982;83: $430-4$.

18 Kaftori JK, Pery M, Kieinhaus U. Ultrasonography in Crohn's disease. Gastointest Radiol 1984;9:137-42.

19 Hata J, Haruma K, Suenaga K, et al. Ultrasonographic assessment of inflammatory bowel disease. Am $\mathcal{F}$ Gastroenterol 1992;87:443-7.

20 Sheridan MB, Nicholson DA, Martin DF. Transabdominal ultrasonography as the primary investigation in patients with suspected Crohn's disease or recurrence: a prospective study. Clin Radiol 1993;48:402-4.

21 Pedersen BH, Gronvall S, Dorph S, et al. The value of dynamic ultrasound scanning in Crohn's disease. Scand $\mathcal{F}$ Gastroenterol 1986;21:96972.

22 Schwerk WB, Beckh K, Raith M. A prospective evaluation of high resolution sonography in the diagnosis of of high resolution sonography in the diagnosis of inflammatory bow 190 : $173-82$.

23 Worlicek H, Lutz H, Heyder N, et al. Ultrasound findings in Crohn's disease and ulcerative colitis: a prospective study. $\mathcal{F}$ Clin Ultrasound 1987;15:153-63. 
24 Limberg B. Diagnosis of acute ulcerative colitis and colonic Crohn's disease by colonic sonography. F Clin Ultrasound

25 Kimmey MB, Wang KY, Haggitt RC, et al. Diagnosis of inflammatory bowel disease with ultrasound. An in vitro study. Invest Radiol 1990;25:1085-90.

26 Maconi G, Parente F, Boliani S, et al. Abdominal ultrasound in the assessment of extent and activity of Crohn's disease: clinical significance and implication of bowel wall thickening. Am $\mathcal{F}$ Gastroenterol 1996;91:1604-9.

27 Harvey RF, Bradshaw JM. A simple index of Crohn'sdisease activity. Lancet 1980;i:514.

28 Maconi G, Boliani S, Bianchi Porro G. Ultrasonographic detection of intestinal complications in Crohn's disease. Dig Dis Sci 1996;41:1643-8.
29 Kelly JK, Preshaw RM. Origin of fistulas in Crohn's disease. f Clin Gastroenterol 1989;11:193-6.

30 Kelly JK, Siu TO The strictures, sinuses, and fissures of Crohn's disease. $\dot{f}$ Clin Gastroenterol 1986;8:594-8.

31 Kelly JK, Sutherland LR. The chronological sequence in the pathology of Crohn's disease. $\mathcal{F}$ Clin Gastroenterol 1988;10: 28-33.

32 Bernstein CN, Boult IF, Greenberg HM, et al. A prospective randomized comparison between small bowel enteroclysis and small bowel follow-through in Crohn's disease. Gastroenterology 1997;113:390-8.

33 Barker DJ, Rose G. Epidemiology in medical practice. 3rd edn. Edinburgh: Churchill Livingstone, 1984.

34 Gasche C, Schober E, Turetschek K. Small bowel barium studies in Crohn's disease. Gastroenterology 1998;114:1349. 